continued fall in the value of money and rise in remuneration in other professions necessitated a further review of academic salaries in 1954, as a result of which the basic salary for non-medical professors was raised from the figure of $£ 1,600$ fixed in 1949 to $£ 1,900$, while for readers and senior lecturers a range of selaries up to $£ 1,850$ was provided in place of the previous $£ 1,600$. The scales for lecturers were raised from $£ 650$ to $£ l, 350$ and for assistant lecturers from $£ 550$ to $£ 650$. The additional recurrent grant for this purpose made it possible to give immediate increases averaging about 17 per cent. After consultation with the universities $\mathbf{a}_{\mathbf{b}}$ scheme was devised, and approved by the Treasury and Inland Revenue Department, under which a supplementary pension can be given where the best single annuity purchasable with the proceeds of the policies held on behalf of the retiring member of staff is less than a specified rate depending on his position and length of university service.

Recently a further increase in the salaries of university teachers has been announced in the House of Commons by the Chancellor of the Exchequer. In a written reply to a question on March 12, he said that from August 1 the salary range for non-medical professors would be $£ 2,300-£ 3,000$; that for readers and senior lecturers up to $£ 2,250$; that for lecturers $£ 900-£ 1,650$, and that for assistant lecturers $£ 700$ $£ 850$.

Discussing technological development, the report recapitulates arguments and decisions already familiar to readers of Nature, and the section dealing with non-recurrent grants also summarizes the recommendations of the Gater Committee and the decisions regarding the expansion of the Imperial College of Science and Technology. Non-recurrent grants approved during the past four years, excluding capital grants of more than $£ 1.5$ million for the expansion of the Imperial College, totalled $£ 23.8$ million. Of this sum, $£ 18,972,000$ was for buildings and $£ 2,199,000$ for sites and properties, the amount of new building work approved totalling about $£ 17 \cdot 25$ million and general expenditure about $£ 16 \cdot 25$ million, bringing the total spent on university building in the past nine years to about $£ 31.25$ million. If, however, the universities could have built at the rate estimated in 1947 as necessary to meet their admitted needs at 1947 prices, their building expenditure should have amounted to about $£ 92$ million. Visits to all the universities and university colleges have impressed the Committee with the extent of overcrowding and congestion, and although the more generous provision of capital grants for buildings to be started in 1957 and later years, rising to $£ 12$ million in 1958, will ease this situation, a steady concentration of building effort of much greater volume than in $1952-56$ is required to realize the desirable expansion of universities. Of major building projects approved in the period, including that at the Imperial College, 7 per cent is for arts, 62 per cent for science and technology, and 31 per cent for libraries, student unions, refectories, halls of residence and the like.

The aggregate income of the universities rose from $£ 25 \cdot 75$ million in 1951-52 to $£ 36 \cdot 89$ million in $1955-56$ and aggregate expenditure from $£ 26 \cdot 13$ million to $£ 38.07$ million, the percentage of income from Parliamentary grants increasing from $66 \cdot 5$ to $72 \cdot 7$. That from fees fell from 14.8 per cent to 10.8 per cent, from endowments from $5 \cdot 2$ to $3 \cdot 8$ per cent, from local authority grants from $4 \cdot 1$ to $3 \cdot 1$ per cent and from donations and subscriptions from 1.9 to 0.9 per cent. Besides the recurrent grant for general purposes, including development in science and technology, totalling $£ 24,883,000$ for $1956-57$, recurrent grants for salary supplementation totalled $£ 2,383,000$; for pension supplementation $£ 67,000$; for the further expansion of technological education $£ 570,000$; and for the Institutes of Education at Liverpool and Reading £25,000. The Committee of Vice-Chancellors and Principals has framed proposals aiming at a greater uniformity of fees as between institutions and

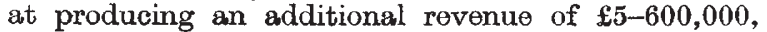
which have been adopted by the universities and will come into force in 1957-58. The Committee believes that the way in which the universities have met the economic strains of the past five years should strengthen public confidence in their financial administration.

\section{PLANT-PARASITIC NEMATODES}

WHE Linnean Society held a symposium on "Plant-parasitic Nematoda" on March 21, with the president in the chair. Introducing the symposium, Prof. B. G. Peters (Imperial College of Science and Technology) briefly outlined the characteristics of the group, pointing out that, although a pointed, hollow, protrusible spear or mouth-stylet had been independently evolved twice among the nematodes, most of the plant parasites were tylenchs. There were various types of association between plants and nematodes, with varying degrees of dependency; also, the complex inter-relationships between parasite and host could be studied in many ways, three of which would be discussed by later speakers. Since two of these papers concerned species of Heterodera, he explained how these cyst-forming species lent themselves to quantitative estimates of population density.

N. G. Hague and J. J. Hesling gave a joint paper on population studies on cyst-forming nematodes, based on work with Heterodera rostochiensis at the Imperial College and with $H$. major at Rothamsted, respectively. By growing plants in pots of soil to which had been added cysts so as to form a geometric series of population densities, they studied the effects of increasing density not only on plant growth and yield, but also on the final population density itself. Thus, both rate of larval multiplication and mean size of cysts decreased with increasing initial density. There was a Gaussian relation between the logarithm of the initial density and the multiplication rate expressed as a proportion of estimated maximum rate.

For the potato root eelworm, decreasing multiplication rate with increasing initial density leads to a maximum final density; any larger initial density then gives a smaller final density. Therefore, unless great care is taken, experiments in which results are assessed by final densities may be completely misleading.

A different approach to host-parasite relationships in Heterodera was outlined by 'T. D. Williams in a paper on "Potatoes Resistant to Root Eelworm". In this he summarized both the recent work of the plant breeders, and his own investigations, at the School of Agriculture, Cambridge, on the influence of resistant strains on the hatching and root-penetration of $H$. rostochiensis larvæ.

An examination of the extensive Commonwealth Potato Collection by Ellenby led to his discovering 
resistance in certain clones of Solanum andigenum (a tuber-forming tetraploid close to $S$. tuberosum) and in the species $S$. vernei (a wild diploid). Repeated back-crossing of the andigenum $\times$ tuberosum hybrid had led to very promising results, but these hybrids produced an active 'hatching factor' and the resultant hatched larvæ readily penetrated the roots, where they stimulated the formation of giant cells just as in normal potatoes. Although only males completed their development in hybrid roots, so that an eelworm population would be rapidly reduced by a succession of these crops, nevertheless this mass invasion of the roots led to a reduced yield of tubers. Worse, there was now evidence of an eelworm strain to which these hybrids were not resistant. Thus, the (at first) less-promising diploid, $S$. vernei, might in the long run prove the more reliable source of resistance.

In the third paper, R. S. Pitcher and J. E. Crosse (East Malling) discussed a very different eelworm, the ectoparasitic leaf-eelworm, A phelenchoides ritzemabosi, which causes trouble in strawberries and other plants. Some years ago, these two workers showed that this eelworm could, in conjunction with Corynebacterium fascians, cause a curious condition called cauliflower disease. The interest of the phenomenon lay in the fact that both pathogens were required in order to get the very typical cauliflower symptoms in the strawberry plant. On the present occasion, Dr. Pitcher went through the subsequent experimental work which was necessary to prove the duplex origins of the disease. It was not enough to have the four categories: with and without eelworm, with and without bacterium (giving either, both, and neither), since in fact wild strains of the bacterium kept vitiating results. In the end, strawberry seedlings had to be grown from sterilized seed by sterile culture methods and eelworms had to be rendered sterile also. It was then possible to show that each pathogen could cause a characteristic symptom, but only together would they produce cauliflower disease.

The ensuing discussion had the effect of underlining the patchwork nature of present knowledge in this field. The plant-parasitic nematodes are not only of great academic interest, readily lending themselves to experimental methods of investigation; they are also of considerable economic importance.

B. G. Peters

\section{A NEW MICROWAVE AMPLIFIER}

DROF. C. H. TOWNES and his associates at Columbia University described in 1955 (Phys. Rev., 99, 1264) a new type of microwave amplifier for which they coined the name 'maser'-microwave amplification of stimulated emission of radiation. The amplification is produced by stimulated emission of radiation from systems in which a higher energy level is more densely populated than a lower one. Townes first demonstrated such an amplifier using molecular beams in gases. Attempts to apply the principle to solids have until very recently met with only partial success.

Last October, N. Bloembergen (Phys. Rev., 104, 324 ; 1956) directed attention to the possible use of power saturation of one transition in a multiple energy level system to obtain a change of sign of the population between another pair of levels. Now paramagnetic substances are characterized by an unpaired spinning electron and an inversion of the spin levels can be obtained. The particular group known as 'ionically bound paramagnetic salts' are very suitable, and Bloembergen showed theoretically that crystals of nickel fluosilicate or gadolinium ethyl sulphate at liquid helium temperatures would be satisfactory to use for a low noise microwave amplifier, or frequency converter, that is, for a solid state 'maser'.

The first successful operation of a 'maser' of this type, using a crystal of lanthanum ethyl sulphate containing about 0.5 per cent $\mathrm{Gd}+++$ and 0.2 per cent $\mathrm{Ce}^{+++}$in contact with liquid helium at $1.2^{\circ} \mathrm{K}$., was demonstrated by H. E. D. Scovil, G. Feher and H. Seidel at the Bell Telephone Laboratories, Murray Hill, New Jersey, on November 27, and a brief description has since been published (Phys. Rev., 105,762 ; 1957). The magnetically dilute para. magnetic salt crystal must have at least three energy levels the transitions of which fall within the microwave frequency range, and the basic operation can be explained by considering these three different energy levels for the unpaired spinning electrons in the crystal lattice for a large applied magnetic field. Normal electron spin states are such that the number occupying state 1 (the lowest energy state) exceeds the number in state 2, which in turn exceeds the number in state 3 (the highest energy state). By irradiating the crystal with sufficient microwave power of the proper frequency, transitions from state 1 to state 3 take place until the populations of these two states are essentially equal (power saturation). Under these conditions, the population of state 2 can be made greater than that of state 1 , and if another, smaller, microwave signal of frequency corresponding to the energy difference between the states 2 and 1 is applied, stimulated transitions will occur, giving rise to radiation with consequent power gain.

In the experimental 'maser' a saturating magnetic field at $17.52 \mathrm{kMc} / \mathrm{s}$. was used to induce transitions between the $-5 / 2$ (the lowest) and the $-1 / 2$ (the highest) energy states in the ground state of the gadolinium ion, and a signal at $9.06 \mathrm{kMc}$./s. was applied between the $-5 / 2$ and the $-3 / 2$ states. The crystal was mounted in a wave-guide cavity which was simultaneously resonant to the two frequencies, and occupied about 8 por cent of the cavity volume, being located at the point of maximum field intensity. Low-temperature operation is necessary in order that power saturation of the crystal takes place for a reasonable amount of energizing oscillator power and also to increase the population difference between the energy levels. For the required separation of the electron spin energy levels a d.c. magnetic field of 2,850 oersteds was applied at an angle of $17^{\circ}$ to the normal to the crystal, but this magnetic field may be altered to change the electron spin energies and thus tume the frequency of the stimulated radiation. The graph of output power at $9 \mathrm{kMc} / \mathrm{s}$. against input power at $17.5 \mathrm{kMc} / \mathrm{s}$. shows an output of 15 microwatts for an input of 200 milliwatts, but the particular merit of the solid state 'maser' is its very low inherent noise (corresponding to thermal noise of $5-10^{\circ} \mathrm{K}$.) compared with ordinary electronic oscillators or amplifiers which depend on the motion of charged particles at high temporatures.

It should be possible with this device to amplify extremely weak radio signals-signals which may be several hundred times weaker than those at present usable-and with proper choice of solid-state materials and operating conditions, the solid-state 'maser' should be a valuable pre-amplifier in a wide variety of microwave applications. $\quad$ S. WEINTroub 